\title{
Chronic kidney disease in Asia - Protocol for a collaborative overview
}

Asian Renal Collaboration (ARC)

Writing committee: Thaminda Liyanage ${ }^{1,2}$, Toshiharu Ninomiya ${ }^{1}$, Vlado Perkovic ${ }^{1}$, Mark Woodward $^{1}$, Heide Stirnadel-Farrant ${ }^{3}$, Kunihiro Matsushita ${ }^{4}$, Kunitoshi Iseki ${ }^{5}$, Hooi Lai Seong ${ }^{6}$, Helen Monaghan ${ }^{1}$ and Vivekanand $\mathrm{Jha}^{7,8}$

1. The George Institute for Global Health, School of Medicine, University of Sydney NSW, Australia

2. Armadale Kelmscott Memorial Hospital, South Metropolitan Health Service Perth, WA, Australia

3. GlaxoSmithKline, Global Epidemiology, Middlesex, United Kingdom

4. Johns Hopkins Bloomberg School of Public Health, Department of Epidemiology Baltimore, USA

5. University Hospital of the Ryukyus, Department of Renal Medicine, Uehara, Nishihara, Okinawa, Japan

6. Sultanah Aminah Hospital, Haemodialysis Unit, Jalan Skudai Johor Bahru, Malaysia

7. The George Institute for Global Health, India, New Delhi, India

8. University of Oxford, Oxford, U.K. 


\section{Corresponding author / contact for reprint requests:}

Vivekanand Jha

The George Institute for Global Health - India

219-221, Splendor Forum

Plot No 3, Jasola District Centre

New Delhi 110025

India

Email: vjha@georgeinstitute.org.in

Phone: +91 114158 8091-93

Fax: +91 1141588090

Running title: Chronic Kidney Disease in the Asia Pacific Region

Word count (Excluding abstract, figures, tables, appendices and references): 2015

Abstract word count: 249

Appendix: 2

Number of tables: 2

Number of figures: 2 


\section{$\underline{\text { ABSTRACT }}$}

\section{Backqround}

The burden of chronic kidney disease (CKD) is growing rapidly around the world. However, there is limited information on the overall regional prevalence of $\mathrm{CKD}$, as well as the prognostic implications and treatment patterns in Asian region. We have established the Asian Renal Collaboration (ARC) with the goal of consolidating region-wide data regarding CKD.

\section{Design and Methods}

This collaborative project will synthesize data and perform meta-analyses of observational studies conducted in Asia. Studies will be identified through a systematic literature search including abstracts, proceedings of meetings, electronic databases such as MEDLINE and EMBASE. Personal enquiry among collaborators and experts in the region will identify additional studies, or other data sources such as registries. Both cross-sectional and longitudinal studies that describe the prevalence of CKD and its complications will be included, as will longitudinal studies that describe important clinical outcomes for people with CKD. Individual participant data will be sought, where possible, from each of the studies included in the collaboration for baseline parameters and subsequent outcomes, in order to maximize flexibility and consistency of data analyses.

\section{Conclusions}

This study is an initiative offering a unique opportunity to obtain information about the prevalence and manifestations of CKD in Asia, as well as its risk factors. The ARC will also provide insights into important outcomes including progression of CKD, CKD complications, cardiovascular disease and death. These findings will improve our understanding of kidney 
disease in Asia, and thus help inform service provision, preventive care and further research across the region.

\section{$\underline{\text { Keywords }}$}

Asia, Cardiovascular disease, chronic kidney diseases, cohort study, prognosis, risk factors 


\section{BACKGROUND}

Chronic kidney disease (CKD) is an important cause of morbidity and mortality around the world. It has been suggested that the incidence and prevalence of CKD and end stage kidney disease (ESKD) will rise sharply in the coming decades, predominately in Asia (1-4). Individuals with CKD have a reduced life expectancy, and those who progress to end-stage kidney disease (ESKD) have 20-fold higher mortality rates compared with age- and sex-matched individuals with normal kidney function (5). The rising number of patients initiating renal replacement therapy in most Asian countries $(1,4,6)$ is placing a substantial burden on healthcare systems.

During the past few decades, epidemiological studies have provided much valuable information about CKD, its progression to ESKD and its associations with complications such as anaemia, cardiovascular disease and mortality. Cross-sectional data indicate that the prevalence of CKD in the general population varies between 6 and 20\% (1, 7-11). However, most of the evidence has been generated from studies conducted in European and North America and less evidence has been derived from Asian populations. Disease patterns and distribution of risk factors differ markedly in many Asian compared to non-Asian populations, as well as varying substantially within the region itself (for example, regional variations in the incidence of IgA nephropathy, CKD secondary to herbal therapy and of undetermined aetiology, lower blood cholesterol and body mass index, younger age of onset, amongst others) (12-16). Even less is known about the factors that determine progression and development of complications amongst subjects with CKD in the region. Therefore, it is imperative that information specific to Asian populations is collected and analyzed to improve our understanding of the challenges, in order to more effectively manage this complex condition in the region. 
Collaborative overviews (with meta-analysis where appropriate), in which data from a number of observational studies are combined (17-21), provide useful information which is additional to that provided by the studies individually. The large number of participants will also facilitate discernment of important differences in the magnitude of associations between disease stages, geographical regions, and between patient subgroups.

The Asian Renal Collaboration (ARC) has been set up to enable these analyses using (where possible) individual participant data from observational studies or other sources, such as registries, in Asia on a broad range of risk factors and clinical outcomes. The main objectives of this project are to obtain information from a large number of subjects about the prevalence, manifestations and risk factors of CKD in Asia. Additionally, this study aims to provide insight into important clinical endpoints including progression of CKD, CKD complications such as anaemia, cardiovascular disease, mineral and bone disorder and mortality. It may be possible to produce estimates of the likely effects on CKD of exposure levels of risk factors. Furthermore, we may be able to estimate the impact of initiatives aiming to prevent CKD and/or its complications on disease progression or other outcomes such as cardiovascular disease. This report describes the protocol for the ARC project.

\section{OBJECTIVES}

We aim to understand the epidemiology and to estimate the impact of CKD in Asia by pooling already existing individual participant data (where available), or summary level data. Therefore, this project has the following main aims: firstly, to estimate the prevalence of CKD in Asian populations, both overall and country-specific; secondly to evaluate the prevalence of risk factors, complications and patterns of treatment in CKD patients in Asia, and thirdly to investigate clinical outcomes in CKD patients in Asia. 
Page 7 of 28 


\section{DESIGN AND METHODS}

\section{Study eligibility}

Studies are potentially eligible for inclusion in this project if they include a population from the Asia-Pacific region (defined as South Asia, Southeast Asia and East Asia according to the United Nations), involve at least 500 adult (18 years or older) participants recorded or planned and have one of the following study designs:

a) Cross-sectional study (or baseline data from longitudinal studies) that describe the prevalence of CKD in a community-based population and/or the prevalence of risk factors, complications and the patterns of treatment in people with CKD

b) Longitudinal studies that describe clinical outcomes of interest in people with CKD

Given the relative paucity of data, we will include all available studies without a time limit.

\section{Identification of studies}

Studies have been identified through a systematic search of the literature including abstracts and proceedings of meetings, as well as electronic databases such as MEDLINE and EMBASE. In addition, personal enquiry among collaborators and experts in the region has identified studies that have not yet been reported. Individual participant data will be sought from each collaborating study (see Appendix 1). Eligible studies identified subsequent to the preparation of this protocol will be invited to participate in the collaboration and to contribute data to the overviews.

\section{Principal risk factors}

The data requested for each participant include: date of baseline survey, date of birth or age at baseline, gender, ethnicity, occupation, education, history of stroke and coronary heart 
disease. The principal risk factors about which data will be sought are as follows: systolic and diastolic blood pressure, diabetes, serum total cholesterol, HDL and LDL cholesterol, triglycerides, obesity (assessed from height and weight), electrocardiogram abnormality, smoking status, alcohol consumption, exercise, serum creatinine, estimated glomerular filtration rate (eGFR), presence of albuminuria or proteinuria, haemoglobin, iron status, calcium, phosphate, parathyroid hormone and Vitamin D levels. For the risk factors measured on multiple occasions, data on all recorded measurements will be sought. Erythropoietin stimulating agent use, iron therapy, anti-hypertensive medications, antithrombotic medications, cholesterol lowering drugs, phosphate binders, calcium, vitamin D, and calcimimetic use will be collected as the information on treatment.

\section{Principal outcomes}

Data will be sought from each study on the date of occurrence of each of the outcomes listed in table 1. CKD stage will be defined according to KDIGO guidelines. ESKD will be defined as receiving maintenance dialysis or having a functioning renal transplant whereas anaemia will be defined as haemoglobin $(\mathrm{Hb})<100 \mathrm{~g} / \mathrm{l}$ or on ESA therapy. Precise details of the diagnostic criteria used for defining the outcomes (eg. major cardiovascular events), and data on the completeness of follow-up will also be collected for individual studies. It is anticipated that definitions for outcomes may vary across included studies, but will be standardized whenever possible. All analyses will be based on events classified according to the tenth revision of the International Classification of Diseases (ICD-10) or similar. Quality assessment of outcome ascertainment will be conducted and subgroup analyses will take place in order to assess for potential bias. For any study that did not use this classification system, events are re-coded by the project secretariat using all available information. 


\section{Data transfer and checking}

A standardised central data request form has been developed to collect the relevant data from each of the involved studies. Data may be provided to the coordinating centre in any format convenient to the collaborator. A unique, anonymous identifier will be sought for each individual in submitted data sets, so as to allow further data linkage should this be necessary in the future. All data provided to the coordinating centre will be carefully checked for completeness and consistency. Computer-generated reports from the data will be referred back to the principal investigator of each study for review and confirmation in order to ensure that the individual study results are incorporated correctly into the overview. All data queries will be discussed and resolved with the responsible collaborating investigator. Where individual participant data are not able to be shared, tabular data will be sought using consistent definitions and statistical code wherever possible.

The data provided for inclusion in the ARC will be held in strict confidence by the coordinating centre. The data manager in the coordinating centre will store all data securely. The data from each study will remain the sole property of the principal investigators of that study and will not be used for any presentation or publication without the consent of the collaborating investigators from that study.

\section{Statistical analysis}

We use cross sectional data. With IPD we obtain prevalence as the relative frequency and compute at least nation specific results. Relevant information will be extracted from published data including by contacting authors if necessary. If we have enough values from published data we will also compute age and sex specific results from the IPD. Prevalence data will be collected from nationally representative studies where these are available, but non-representative national and regional data will also be collected where these are not available. Summary pooled estimates will be calculated, and associations with risk factors 
estimated. Models will be developed in an effort to estimate CKD prevalence where data are not available. In the first instance, the principal analyses of the associations of risk factors with disease for each study will be performed using Cox proportional hazards models (Cox 1972). We shall pool results using random effects meta-analysis, weighting by inverse variance (22). If appropriate, we will adjust for the effects of regression dilution bias using repeat risk factor measurement data (ESCHDC group 1998). Analyses will also be adjusted for potential confounding factors and stratified by potential effect modifiers. Analyses will be conducted to assess the separate associations in men and women, in different regions and ethnic groups, in different age groups (at baseline and at death), and in different periods of follow-up. Analyses will be conducted to determine whether any subgroup differences are due to confounding (eg. different age distributions in the cohorts), effect modification (eg. different predictor-risk relationship across regions) or different diagnostic practices between countries, using random effects meta-regression(22). In addition to exploring heterogeneity of the associations between these subgroups, we will explore heterogeneity between individual studies (adjusting for major confounders) using i-squared statistics and chi-square tests(22). Sensitivity analyses taking account of factors including completeness of follow-up, methods of outcome assessment, availability of repeat risk factor measurements and imputation of missing values(22) will be performed to determine the robustness of the findings. This analysis plan will be continuously evaluated and a more detailed analysis plan will be developed, depending on available data.

\section{Time frame and publication policy}

Initial data collation is expected to be completed by the end of December 2015. Statistical analysis will begin in 2015 with reports prepared for publication from 2015 onwards. The results of the study will be disseminated by manuscripts in peer-reviewed publications and by presentations at international meetings. All reports from this project will be sent to all 
collaborating investigators for prior comments and approval. All such reports will be published in the name of the Asian Renal Collaboration. Ethical approval and subject consent

Individual studies will have obtained ethical approval and subject consent from their relevant institutions prior to participation in the collaboration. Thus, no further ethical approval or subject consent will be sought from individual participants.For the process of central data collection and analysis, a separate ethics approval has been granted by the University of Sydney, Australia (ARC project ID: 2015/217).

\section{ORGANISATION}

The ARC is a collaborative project between the principal investigators of contributing observational studies. The project secretariat is based at the George Institute for Global Health, Sydney, Australia. The Executive Committee and the Secretariat, both of which are responsible to the collaborating individual study investigators, manage the central coordination of the overall project. External researchers will be able to propose research projects to ARC. The Steering Committee comprises regional representatives (from China, Japan, South Korea, India, Taiwan, Thailand and Malaysia) and representatives from the project secretariat (appendix 2, figure 1) and has overall responsibility for the design, conduct, progress, data collection, data analysis and publication of study results.

More than 20 studies from 7 countries are participating to date, representing more than 2.1 million study participants. This project has been funded by a grant from GlaxoSmithKline limited (table 2, figure 2). 


\section{CONCLUSION}

The ARC will aim to define the burden of CKD in Asia, and identify associated patters of risk factors and outcomes. It will highlight differences and similarities between CKD in Asia and other regions, and provide important information that will inform future research directions in the region, as well as health service planning. 


\section{ACKNOWLEDGMENT}

Financial support: This project is supported by GlaxoSmithKline Limited. 


\section{REFERENCES}

1. United States Renal Data System - 2012 Atlas of CKD \& ESRD. 2012.

2. Lozano R, Naghavi M, Foreman K, Lim S, Shibuya K, Aboyans V, et al. Global and regional mortality from 235 causes of death for 20 age groups in 1990 and 2010: a systematic analysis for the Global Burden of Disease Study 2010. Lancet. 2012;380(9859):2095-128. 3. Jha V, Garcia-Garcia G, Iseki K, Li Z, Naicker S, Plattner B, et al. Chronic kidney disease: global dimension and perspectives. Lancet. 2013;382(9888):260-72.

4. Liyanage T. Worldwide access to treatment for end-stage kidney disease: a systematic review. Lancet. 2015 May 16;385(9981):1975-82.

5. Lowrie EG, Lew NL. Death risk in hemodialysis patients: the predictive value of commonly measured variables and an evaluation of death rate differences between facilities. American journal of kidney diseases : the official journal of the National Kidney Foundation. 1990;15(5):458-82.

6. Nakai S, Watanabe Y, Masakane I, Wada A, Shoji T, Hasegawa T, et al. Overview of regular dialysis treatment in Japan (as of 31 December 2011). Therapeutic apheresis and dialysis : official peer-reviewed journal of the International Society for Apheresis, the Japanese Society for Apheresis, the Japanese Society for Dialysis Therapy.

2013;17(6):567-611.

7. Chadban SJ, Briganti EM, Kerr PG, Dunstan DW, Welborn TA, Zimmet PZ, et al. Prevalence of kidney damage in Australian adults: The AusDiab kidney study. Journal of the American Society of Nephrology : JASN. 2003;14(7 Suppl 2):S131-8.

8. Chen J, Wildman RP, Gu D, Kusek JW, Spruill M, Reynolds K, et al. Prevalence of decreased kidney function in Chinese adults aged 35 to 74 years. Kidney international. 2005;68(6):2837-45.

9. White SL, Cass A, Atkins RC, Chadban SJ. Chronic kidney disease in the general population. Advances in chronic kidney disease. 2005;12(1):5-13.

10. Tanaka H, Shiohira Y, Uezu Y, Higa A, Iseki K. Metabolic syndrome and chronic kidney disease in Okinawa, Japan. Kidney international. 2006;69(2):369-74.

11. Perkovic V, Cass A, Patel AA, Suriyawongpaisal P, Barzi F, Chadban S, et al. High prevalence of chronic kidney disease in Thailand. Kidney international. 2008;73(4):473-9. 12. Kesteloot H, Huang DX, Yang XS, Claes J, Rosseneu M, Geboers J, et al. Serum lipids in the People's Republic of China. Comparison of Western and Eastern populations. Arteriosclerosis (Dallas, Tex). 1985;5(5):427-33.

13. Group IMC. Risk factors for cardiovascular disease in the developing world. A multicentre collaborative study in the International Clinical Epidemiology Network (INCLEN). . Journal of clinical epidemiology. 1992;45(8):841-7.

14. Abraham G, Varughese S, Thandavan T, lyengar A, Fernando E, Naqvi SA, et al. Chronic kidney disease hotspots in developing countries in South Asia. Clinical kidney journal. 2016;9(1):135-41.

15. Rajapurkar MM, John GT, Kirpalani AL, Abraham G, Agarwal SK, Almeida AF, et al. What do we know about chronic kidney disease in India: first report of the Indian CKD registry. BMC nephrology. 2012;13:10.

16. Vachvanichsanong $P$, Dissaneewate $P$, McNeil E. Childhood chronic kidney disease in a developing country. Pediatric nephrology (Berlin, Germany). 2008;23(7):1143-7.

17. MacMahon S, Peto R, Cutler J, Collins R, Sorlie P, Neaton J, et al. Blood pressure, stroke, and coronary heart disease. Part 1, Prolonged differences in blood pressure: prospective observational studies corrected for the regression dilution bias. Lancet. 1990;335(8692):765-74.

18. Law MR, Wald NJ, Wu T, Hackshaw A, Bailey A. Systematic underestimation of association between serum cholesterol concentration and ischaemic heart disease in observational studies: data from the BUPA study. BMJ (Clinical research ed).

1994;308(6925):363-6. 
19. O'Seaghdha CM, Perkovic V, Lam TH, McGinn S, Barzi F, Gu DF, et al. Blood pressure is a major risk factor for renal death: an analysis of 560352 participants from the Asia-Pacific region. Hypertension. 2009;54(3):509-15.

20. Matsushita K, van der Velde M, Astor BC, Woodward M, Levey AS, de Jong PE, et al. Association of estimated glomerular filtration rate and albuminuria with all-cause and cardiovascular mortality in general population cohorts: a collaborative meta-analysis. Lancet. 2010;375(9731):2073-81.

21. Matsushita K, Ballew SH, Astor BC, Jong PE, Gansevoort RT, Hemmelgarn BR, et al. Cohort profile: the chronic kidney disease prognosis consortium. International journal of epidemiology. 2013;42(6):1660-8.

22. Woodward (2014) Epidemiology: Study Design and Data Analysis. Third edition. CRC Press, Boca Raton. 2014. 
Steering committee: Vivekanand Jha (India-Chair), Vlado Perkovic (Australia-Deputy Chair), Atsushi Wada (Japan), Daiki Inaguma (Japan), Heide Stirnadel-Farrant (United Kingdom), Helen Monaghan (Australia), Ho Jun Chin (South Korea), Hong Zhang (China), Hooi Lai Seong (Malaysia), Ikuto Masakane (Japan), Kamal Shah (India), Kazuhiko Tsuruya (Japan), Kearkiat Praditpornsilpa (Thailand), Kunihiro Matsushita (USA), Kunitoshi Iseki (Japan), Luxia Zhang (China), Mariko Miyazaki (Japan), Mark Woodward (Australia), Masafumi Fukagawa (Japan), Ming-Yen Lin (Taiwan), Rajasekara Chakravarthi (India), Satoshi Ogata (Japan), Shang-Jyh Hwang (Taiwan), Takayuki Hamano (Japan), Thaminda Liyanage (Australia), Toshiharu Ninomiya (Japan), Yoshinari Yasuda (Japan), Young-Hwan Hwang (Korea), Zuo Li (China) 


\section{FIGURE LEGENDS}

Figure 1: Organisational Structure of the Asian Renal Collaboration

Figure 2: Studies included in the Asian Renal Collaboration (as at 31/11/2015) 


\section{Table 1: Principal outcomes of interest and their ICD-10 codes (where available)}

\section{The prevalence of $C K D$ in Asia}

$\begin{array}{lr}\text { a) Reduced eGFR } & \text { N18 }\end{array}$

$\begin{array}{lr}\text { b) Presence of albuminuria/proteinuria } & \text { R80 }\end{array}$

$\begin{array}{lr}\text { c) CKD stages (defined by KDIGO) } & \text { N18 }\end{array}$

d) ESKD (defined by treated ESKD - dialysis, HD and PD and transplant) N18.5

$\begin{array}{lr}\text { e) Renal transplant recipients } & \text { Z94 }\end{array}$

\section{Prevalence of risk factors, complications and treatment patterns among CKD patients in Asia}

a) Clinical characteristics - Age, gender, cause of CKD, history of blood pressure, diabetes mellitus, BMI, smoking status, other co-morbidities

b) Laboratory characteristics - Haemoglobin, iron stores, albumin, calcium, phosphate, PTH, Vitamin D, urine protein/creatinine ratio, urine albumin

c) Treatment pattern- Iron, ESA, antihypertensive medication, Vitamin D, and other treatments

d) Prevalence of anaemia defined by haemoglobin ( $\mathrm{Hb})<100$ or on ESA therapy

e) Quality of life

\section{Clinical outcomes in CKD patients in Asia}

a) Kidney outcomes
1) Increase in $C K D$ stage
2) Progression to ESKD
3) Doubling of serum creatinine

b) Cardiovascular outcomes

1) Major cardiovascular events (Coronary heart disease, stroke, or cardiovascular mortality)

2) Coronary heart disease (Myocardial infarction or hospitalization from angina)

3) Stroke

4) Cardiovascular mortality

5) Hospitalization from heart failure

6) Any other atherosclerotic disease (e.g. peripheral arterial disease and aneurysm)

7) Thromboembolic events (vascular access and non-vascular access related) 
c) Mortality

1) All-cause mortality

2) Cause-specific mortality

$\begin{array}{ll}\text { All cardiovascular disease } & \text { 100-178 }\end{array}$

$\begin{array}{ll}\text { Coronary heart disease } & 120-125\end{array}$

Pulmonary circulatory disease $\quad$ 126-128

Sudden cardiac death $\quad$ 149-149

$\begin{array}{ll}\text { Heart failure } & 150\end{array}$

$\begin{array}{lr}\text { Stroke } & 160-169\end{array}$

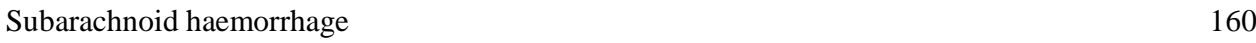

Haemorrhagic stroke $\quad$ 161-162

Ischaemic stroke $\quad$ 163-166

$\begin{array}{ll}\text { All neoplasms } & \text { C00-C97 }\end{array}$

$\begin{array}{ll}\text { Oesophageal cancer } & \text { C00-C14 }\end{array}$

$\begin{array}{ll}\text { Stomach cancer } & \text { C10-C16 }\end{array}$

$\begin{array}{ll}\text { Colorectal cancer } & \text { C18-C21 }\end{array}$

$\begin{array}{ll}\text { Liver cancer } & \text { C22 }\end{array}$

$\begin{array}{ll}\text { Lung cancer } & \text { C33-C34 }\end{array}$

$\begin{array}{ll}\text { Breast cancer } & \text { C50 }\end{array}$

$\begin{array}{lll}\text { Liver disease } & \text { K70-K77 }\end{array}$

$\begin{array}{ll}\text { Kidney disease } & \text { N00-N19 }\end{array}$

$\begin{array}{ll}\text { Lung disease } & \text { J00-J99 }\end{array}$

ICD - 10: Tenth version of the International Classification of Diseases 
Table 2: Individual study characteristics of studies included in the Asian Renal Collaboration (as at 31/11/2015)

\begin{tabular}{|c|c|c|c|c|}
\hline Dataset & Country & No. subjects & Funding & Current status \\
\hline \multicolumn{5}{|l|}{ Studies based on general population } \\
\hline National survey of CKD & China & 50000 & & Tabulated data received \\
\hline Okinawa cohort study & Japan & 154265 & & IPD received \\
\hline Korean general population study & South Korea & 147312 & & IPD received \\
\hline CKD epidemiology survey in southern Taiwan & Taiwan & 3352 & & IPD received \\
\hline Multicenter prospective cohort study & Thailand & 3000 & & Awaited \\
\hline \multicolumn{5}{|l|}{ Studies based on CKD Patients } \\
\hline Chinese Cohort Study of CKD in China & China & 3200 & & Awaited \\
\hline The CKD-JAC Study & Japan & 2977 & & Tabulated data received \\
\hline Nagoya CKD Cohort & Japan & 389 & & Tabulated data received \\
\hline The Hisayama Study & Japan & 3503 & & Tabulated data received \\
\hline The Gonryo Study & Japan & 2692 & & Tabulated data received \\
\hline Korean CKD Cohort Study "KNOW-CKD". & South Korea & ? & & Awaited \\
\hline CKD stage 3-5 Clinical Cohort —Kaohsiung Hospital & Taiwan & 3334 & & Awaited \\
\hline
\end{tabular}

Studies based on ESRD patients

Registry data

Beijing ESRD Registry

China $\quad 12500$

Awaited

Japan Society of Dialysis Therapy Registry

Japan 301538

Malaysian Dialysis and Transplant Registry

Malaysia 32159

Korean Society of Nephrology - ESRD Registry

South Korea 72867

Taiwan ESRD Registry

Taiwan $\quad 75000$

Thailand Renal Replacement Registry

Thailand 52641

Tabulated data received

Tabulated data received IPD received

Awaited

IPD received

Other datasets

Indian Dialysis Cohort - 1

India 1653

India 2926

Indian Dialysis Cohort - 2

Japan 3567

The Q-Cohort Study

Japan

1515

IPD received

IPD received

The (AICOPP) Study

Tabulated data received

IPD: Individual patient level data

Tabulated data received 
Figure 1: Organisational Structure of the Asian Renal Collaboration

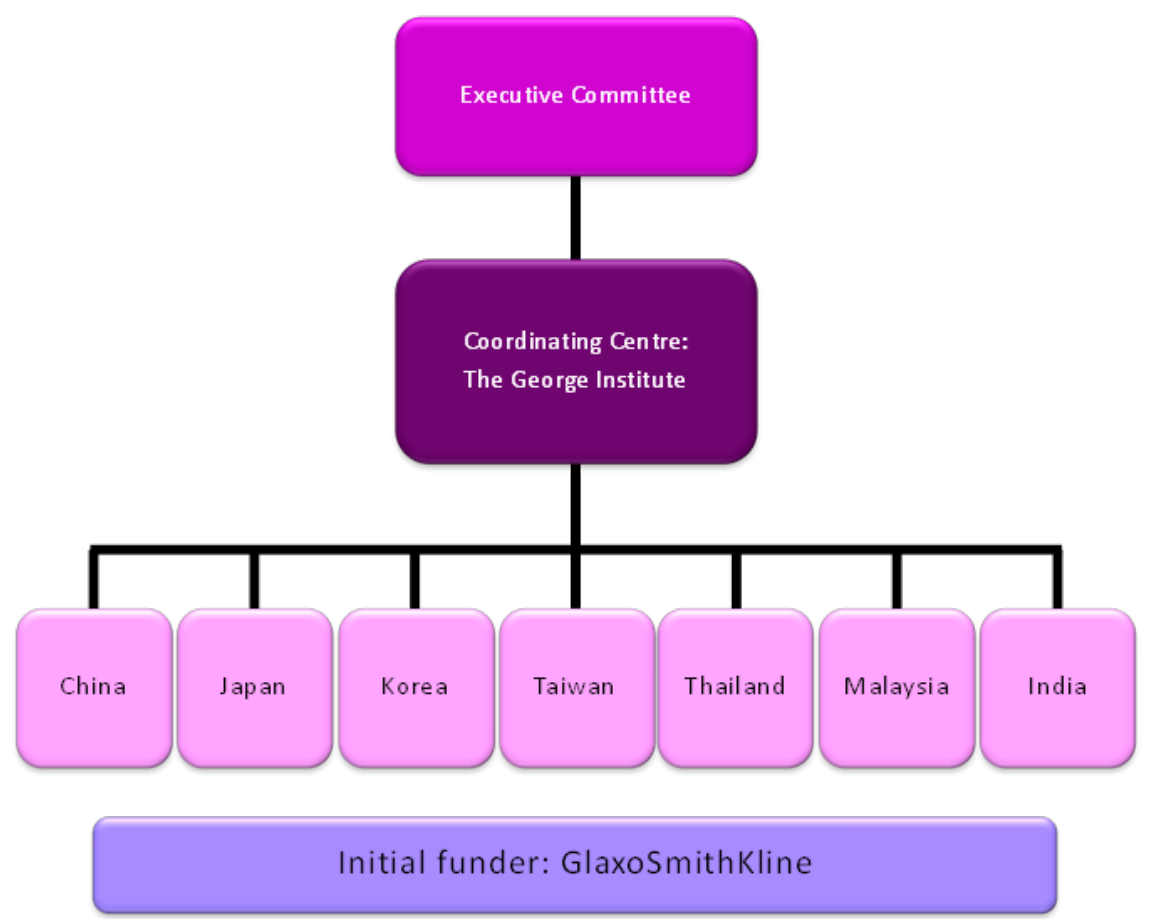


Figure 2: Studies included in the Asian Renal Collaboration (as at 31/11/2015)

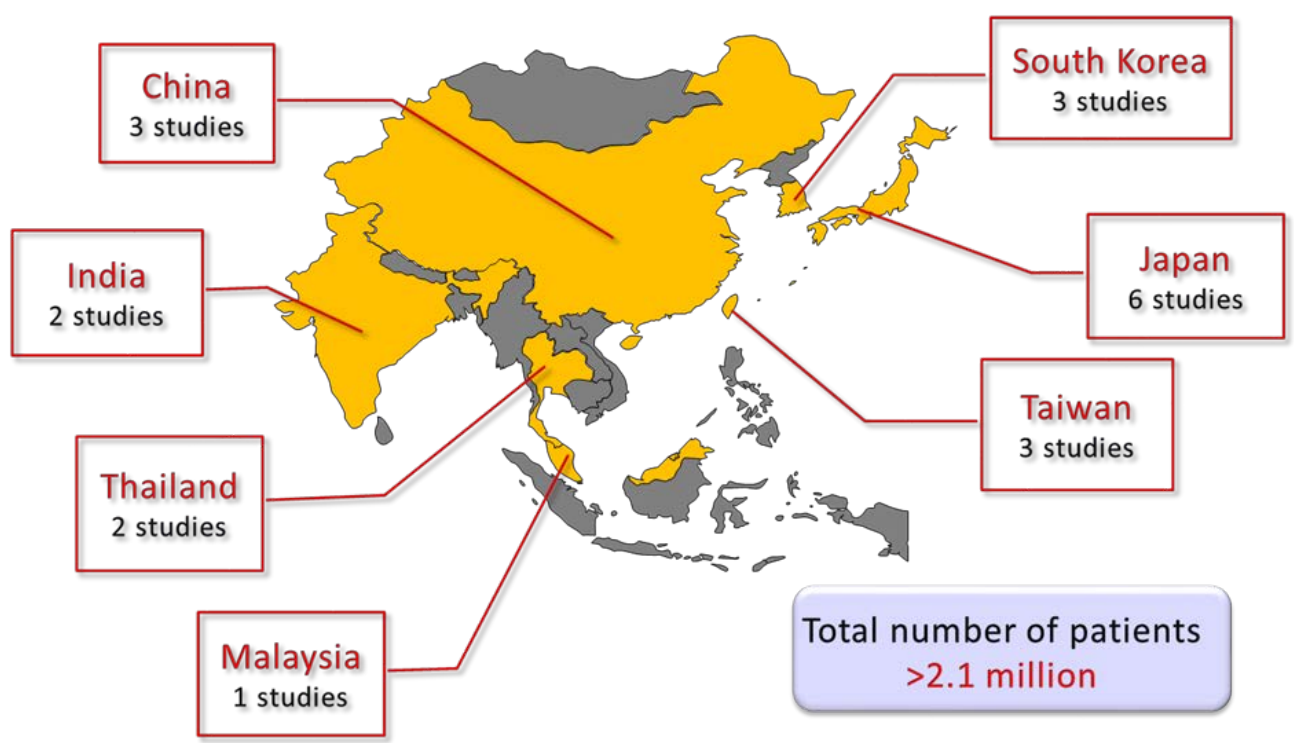




\section{APPENDIX 1: Data Request Format}

\section{Demographic factors}

1.1 Date of birth or age at baseline

1.2 Gender

1.3 Ethnicity

1.4 Occupation (for whole cohort or individually, if available)

1.5 Education level

1.6 Some unique (but anonymous) identifier in case queries arise

\section{Baseline survey data (coded in whatever way the study has used)}

Details of the coding conventions, preferably accompanied by a copy of the questionnaire or data coding or data entry forms used in the study.

2.1 Date or year of the baseline survey

2.2 History of stroke and coronary heart disease

2.3 Cause of kidney disease

2.4 Systolic and diastolic blood pressure

2.5 Diabetes

2.6 Lipid parameter (serum total, HDL- and LDL cholesterol and triglycerides)

2.7 Body parameters (Height, weight, body mass index) 
2.8 Electrocardiogram abnormality (Left ventricular hypertrophy and atrial fibrillation)

2.9 Smoking status

2.10 Alcohol consumption

2.11 Exercise

2.12 Serum creatinine, eGFR

2.13 Urinary albumin-to-creatinin ratio or daily albumin excretion

2.14 Presence of proteinuria, urinary protein-to-creatinne ratio, or daily protein excretion

2.15 Haematology (Haemoglobin, white cell count and platelets)

2.16 Iron status (Serum iron, serum transferrin and serum ferritin)

2.17 Biochemistry (Urea, electrolytes, calcium and phosphate)

2.18 Parathyroid hormone and Vitamin D

\section{Treatment}

3.1 Erythropoietin stimulating agent (yes/no, type, dose, mode of administration)

3.2 Iron therapy (Use or not, type, dose, mode of administration)

3.3 Anti-hypertensive medications (y/n, type, dose)

3.4 Antithrombotic medications (y/n, type, dose)

3.5 Cholesterol lowering drugs ( $\mathrm{y} / \mathrm{n}$, type, dose)

3.6 Phosphate binders (y/n, type, dose) 
3.7 Calcium and Vitamin D therapy (y/n, type, dose)

3.8 Calcimimetics (y/n, type, dose)

\section{Repeat survey(s) data}

Please provide data from any repeat survey(s) in a format as similar as conveniently possible to that for the baseline survey.

\section{Events}

5.1 Date last known to be alive (if not recorded as dead)

5.2 Date of event or, if date not available age at event, for all non-fatal events of these types:

a) Kidney outcomes

1) Increase in CKD stage

2) Progression to ESKD

3) Doubling serum creatinine

b) Cardiovascular outcomes

1) Major cardiovascular events

(Coronary heart disease, stroke, or cardiovascular mortality)

2) Coronary heart disease (Myocardial infarction or hospitalization from angina) 
3) Stroke

4) Cardiovascular mortality

5) Hospitalization from Heart failure

6) Any other atherosclerotic disease (e.g. peripheral arterial disease and aneurysm)

5.3 Date of death (or, age at death, if date not available)

5.4 Underlying cause of death [preferably coded according to some specified version or other (e.g. 7th, 8th, 9th or 10th) of the 4-digit International Classification of Diseases, but if a 4-digit ICD code is not available then whatever code the study already uses]

5.5 Stroke investigated by CT/MRI (y/n)

5.6 Autopsy $(\mathrm{y} / \mathrm{n})$ 


\section{Appendix 2: Committee members and collaborators of the ARC}

\begin{tabular}{|c|c|c|}
\hline Secretariat & Executive committee & Steering committee* \\
\hline Helen Monaghan & Heide Stirnadel-Farrant & Atsushi Wada \\
\hline Mark Woodward & Helen Monaghan & Daiki Inaguma \\
\hline Thaminda Liyanage & Ho Jun Chin & Heide Stirnadel-Farrant \\
\hline Toshiharu Ninomiya & Hong Zhang & Helen Monaghan \\
\hline Vivekanand Jha & Kearkiat Praditpornsilpa & Ho Jun Chin \\
\hline \multirow[t]{24}{*}{ Vlado Perkovic } & Kunitoshi Iseki & Hong Zhang \\
\hline & Luxia Zhang & Hooi Lai Seong \\
\hline & Mark Woodward & Ikuto Masakane \\
\hline & Masafumi Fukagawa & Kamal Shah \\
\hline & Shang-Jyh Hwang & Kazuhiko Tsuruya \\
\hline & Takayuki Hamano & Kearkiat Praditpornsilpa \\
\hline & Thaminda Liyanage & Kunihiro Matsushita \\
\hline & Toshiharu Ninomiya & Kunitoshi Iseki \\
\hline & Vivekanand Jha & Luxia Zhang \\
\hline & Vlado Perkovic & Mariko Miyazaki \\
\hline & Yoshinari Yasuda & Mark Woodward \\
\hline & Zuo Li & Masafumi Fukagawa \\
\hline & & Ming-Yen Lin \\
\hline & & Rajasekara Chakravarthi \\
\hline & & Satoshi Ogata \\
\hline & & Shang-Jyh Hwang \\
\hline & & Takayuki Hamano \\
\hline & & Thaminda Liyanage \\
\hline & & Toshi Ninomiya \\
\hline & & Vivek Jha \\
\hline & & Vlado Perkovic \\
\hline & & Yoshinari Yasuda \\
\hline & & Young-Hwan Hwang \\
\hline & & Zuo Li \\
\hline
\end{tabular}

*Steering committee consists of executive committee members and other collaborators not included in executive committee 